\title{
Three-dimensional stability analysis of maxillomandibular advancement surgery with and without articular disc repositioning
}

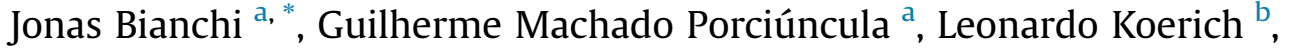 \\ Jaqueline Ignácio a, Larry Miller Wolford ${ }^{\mathrm{c}}$, João Roberto Gonçalves ${ }^{\mathrm{a}}$ \\ a Department of Pediatric Dentistry, São Paulo State University (UNESP), School of Dentistry, Araraquara, SP, Brazil \\ b Private Practice, Charlotte, NC, USA \\ ${ }^{\mathrm{c}}$ Department of Oral and Maxillofacial Surgery, Texas A\&M University Health Science Center, Baylor College of Dentistry, Baylor University Medical Center, \\ Dallas, TX, USA
}

\section{A R T I C L E I N F O}

\section{Article history:}

Paper received 27 October 2017

Accepted 11 May 2018

Available online 18 May 2018

\section{Keywords:}

Orthognathic surgery

Cone-beam computed tomography

Imaging

Three-dimensional

Temporomandibular joint disc

\begin{abstract}
A B S T R A C T
This retrospective cohort study aimed to assess, three-dimensionally, mandible and maxilla changes following maxillomandibular advancement (MMA), with and without repositioning of TMJ articular discs. The sample comprised cone-beam computed tomography data from 32 subjects: group $1(n=12)$ without disc displacement and group $2(n=20)$ with bilateral disc repositioning. An automatic cranial base superimposition method was used to register the images at three time points: T1 (preoperative), T2 (postoperative), and T3 (at least 11 months follow-up). To assess surgical changes (T2-T1) and adaptive responses (T3-T2), the images were compared quantitatively and qualitatively using the shape correspondence method. The results showed that surgical displacements were similar in both groups for all the regions of interest except the condyles, which moved in opposite directions - group 1 to superior and posterior positions, and group 2 to inferior and anterior positions. For adaptive responses, we observed high individual variability, with lower variability in group 2. Sagittal relapse was similar in both groups. In conclusion, there were no significant differences in skeletal stability between the two groups. The maxillomandibular advancement surgeries, with rotation of the occlusal plane, had stable results for both groups immediately after surgery and at 1-year follow-up.
\end{abstract}

๑) 2018 European Association for Cranio-Maxillo-Facial Surgery. Published by Elsevier Ltd. All rights reserved.

\section{Introduction}

Patients with skeletal class II malocclusion and a vertical growth pattern may require orthodontic and surgical correction. Some characteristics, such as increased facial height, mandibular clockwise rotation, narrow upper arch, decreased airway space, and mouth breathing reinforce the need for a surgical-orthodontic approach to restoring the masticatory and respiratory functions, and for improvement of facial aesthetics (Arnett et al., 1996; Wolford, 2003; Borstlap et al., 2004; Goncalves et al., 2013).

Maxillomandibular advancement (MMA) with counterclockwise rotation of the occlusal plane is stable and is the indicated surgical protocol for the treatment of most class II patients.

\footnotetext{
* Corresponding author. Rua Humaitá, 1680, Centro, CEP: 14801-903, Araraquara, SP, Brazil.

E-mail address: jonasbianchi.unesp@gmail.com (J. Bianchi).
}

However, when performed in patients with vertical growth its predictability decreases (Proffit et al., 2007; Franco et al., 2013). Some of the aspects that could lead to instability are the design of the osteotomy, increased load on the TMJs, condylar torques with consequent resorptions, and the system of internal rigid fixation used (Arnett et al., 1996; Gill et al., 2008; Goncalves et al., 2013).

Previous studies have demonstrated an association between patients with vertical growth and increased susceptibility to internal derangement of the temporomandibular joint (TMJ). Orthognathic surgical correction can trigger skeletal relapses, which could relate to the integrity of the articular disc prior to surgery. To maintain a stable and healthy TMJ, open disc repositioning surgery has become a treatment option; however, views on condylar and skeletal stability vary among surgeons and researchers (Arnett et al., 1996; Mehra and Wolford, 2001; Wolford et al., 2003; Gerressen et al., 2007; Gonçalves et al., 2008; Sidebottom, 2009). 
The purpose of this study was to compare, in three-dimensions (3D), the stability of MMA with and without TMJ articular disc repositioning in patients with hyperdivergent profiles.

\section{Materials and methods}

This retrospective study was approved by the ethical committee of São Paulo State University (number: 01125412.2.0000.5416).

The study sample comprised 32 digitized CBCT files from consecutive patients operated on by one surgeon at Baylor University Medical Center, Dallas, TX, USA. The images were analysed in the Pediatric Dentistry department at São Paulo State University (UNESP), School of Dentistry, Araraquara, SP, Brazil.

All patients included in this study had vertical growth patterns (SN.GoMe $>36^{\circ}$ ) and indication for maxillomandibular surgical advancement.

The sample was divided into two groups: group 1 - MMA ( $n=12$ ) individuals with normal TMJ, submitted for maxillomandibular advancement surgery only; and group 2 - MMAd $(n=20)$ individuals with disc displacements on both TMJs, submitted for MMA surgery with simultaneous TMJ disc repositioning (Mehra and Wolford, 2001). Inclusion criteria were: a) patients with normal TMJs without disc displacement confirmed by clinical examination and MRI following Ahmad et al.'s protocol (Ahmad et al., 2009); b) patients with TMJ disc displacements confirmed after MRI assessment using the same protocol; and c) CBCTs taken prior to surgery, immediately after surgery (1-10 days), and at the longest follow-up (average 12.4 and 16.1 months after surgery for MMA and MMAd respectively). Exclusion criteria included: a) history of syndromes, facial trauma, or cleft lip and/or palate; and b) previous TMJ intervention.

The CBCT machine was an I-Cat Platinum (Imaging Science, Hatfield, PA), with the following parameters: scan time $17.8 \mathrm{~s}$, isotropic voxel of $0.3 \mathrm{~mm}$, and a $17 \times 23 \mathrm{~cm}$ field of view. The patients were instructed to be in maximum intercuspal position and after the acquisitions the DICOM (Digital Imaging and Communications in Medicine) files were imported into Dolphin Imaging 11.5 (Dolphin Imaging \& Management Solutions, Chatsworth, Calif). In Dolphin, the lateral cephalogram was traced to confirm the skeletal morphology of patients.

The images were reformatted to an isotropic $0.5 \mathrm{~mm}$, according to Cevidanes et al.'s protocol (Cevidanes et al., 2005). Semi-automatic segmentation of the cranial bases, maxilla, and mandibles were done with ITK-Snap software version 2.2.4 (Yushkevich et al., 2006). Imagine 1.2.1 software (this version is not currently supported, but the new version can be found at http://www.slicer.org/slicerWiki/index.php/Documentation/Nigh tly/Extensions/CMFreg) was used to superimpose the CBCT data using a pre-surgical scan as reference trough the voxel intensity equivalence from the cranial base between the images. Then, measurements were done using the SPHARM-PDM toolbox (Spherical Harmonic - Point Distributed Model) and the shape correspondence method (Paniagua et al., 2011; de Paula et al., 2013; Nguyen et al., 2013). The results were obtained via colorcoded maps, indicating the amount of displacement, and vectors to illustrate the direction of displacement. Two regions of interest were selected in the distal segment of the mandible (chin and B-point), four in the proximal segment (mandibular ramus, and lateral, medial, and posterior poles of the condyles), and one in the maxilla - point A (Fig. 1).

Assessments were performed between T1 and T2 (surgical changes) and between T2 and T3 (adaptive responses and/or displacements). Quantitative analysis corresponded to the largest displacement between two corresponding points on the superficies in each region of interest, measured in millimetres. We also performed a visual qualitative analysis (Table 5) that corresponded to the predominant directions of displacement, with 6 degrees of freedom. To be considered prevalent, the directions of displacement needed to occur in at least $50 \%$ of the individuals in each group. These were classified as follows: three in translational axis (superior/inferior, lateral/medial, anterior/posterior), and three in rotational axis (roll medial/roll lateral, yaw anterior/yaw posterior, pitch up/pitch down).

Statistical analyses were done using the software IBM SPSS Statistics 21.0 (Armonk, NY: IBM Corp). The intraclass correlation coefficient (ICC) was ascertained to assess the reproducibility through measurements that were repeated after 10 days in $20 \%$ of the sample. Student's $t$-test was used to compare the differences between the two types of surgery in each anatomical region. Our main hypothesis was that there were no differences between the two groups in terms of surgical stability.

\section{Results}

Table 1 presents descriptive age and follow-up data, by group and gender. Table 2 shows the cephalometric comparison between groups before (T1) and immediately following surgery (T2-T1), calculated using Student's $t$-test. The results confirm the hypothesis of equality between cephalometric values at T1 and after surgical changes (T2-T1). Table 3 shows the statistical analysis for T2-T1, comparing both groups using Student's $t$-test. Surgical changes were statistically different between the groups in the following areas: Lramus, Lmedpole, Lsupcond, Lpostcond, Rramus, and Rmedpole. Table 4 shows that T3-T2 adaptive responses and/or displacements were statistically equal between the two groups according to Student's $t$-test. The ICC results for reproducibility were higher than 0.97 for all the regions of interest. Figs. 2 and 3 show the percentages of patients per amount of displacement for each group and at each time.

Table 5 shows an observational and descriptive case-by-case analysis (qualitative method). The surgical changes (T2-T1) and the predominant displacements and/or adaptive responses (T3-T2) occurred with 6 degrees of freedom in both groups. Finally, Figs. 4-7 illustrate examples of the main displacements and remodelling that occurred during $\mathrm{T} 2-\mathrm{T} 1$ and $\mathrm{T} 3-\mathrm{T} 2$.

\section{Discussion}

The aim of this study was to evaluate, three-dimensionally, the stability of MMA and the influence of the articular disc repositioning. Simultaneous articular disc repositioning provided similar maxillary and mandibular stability when compared with patients with normal TMJs who underwent MMA. The changes occurring in the T2-T1 interval were interpreted as displacements only because they happened within 10 days of surgery, and this decreased the possibility of adaptive responses, whereas other studies evaluated the T2 CBCTs at the removal of the interocclusal splint or 6 weeks after surgery (Carvalho et al., 2010; de Paula et al., 2013). In T3-T2 those changes could be adaptive responses and/or displacements; however, it is impossible to distinguish these quantitatively with the method used in this study because the image registration was in the cranial base.

Franco et al. (2013), after 3 years of follow up, reported displacements and/or remodelling of the chin in the same direction as our study (inferior and posterior - Fig. 6), but with lower magnitude - up to $2 \mathrm{~mm}$ in $17 \%$ of cases (Franco et al., 2013). This difference probably occurred due to the sample's morphological characteristics, smaller magnitude of advancement, and use of a closest point method with results that are smaller than vectors (De Clerck et al., 2012; Nguyen et al., 2013). In the sagittal direction, 


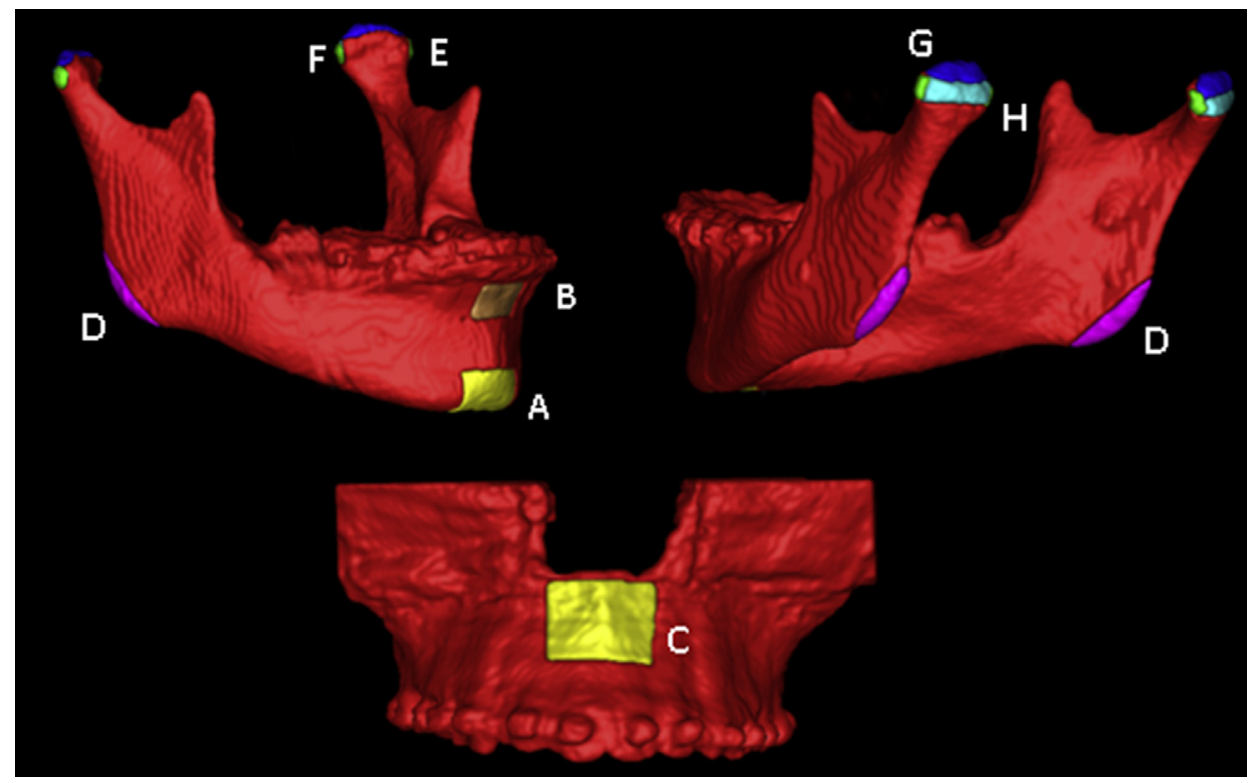

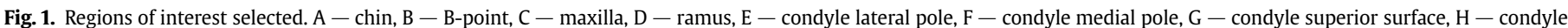
posterior-superior.

both groups showed similar behaviour 11 months after surgery. Otherwise, we noticed that the follow-up analysis (T3-T2) was contrary to the surgical movement performed in T2-T1 (Fig. 6). Also, in the distal segment, as expected, the surgical changes (T2-T1) promoted counterclockwise rotation of the occlusal plane and a decreased mandibular plane angle in both groups. These rotations are the result of predominant displacements in the chin and B-point that moved anteriorly and superiorly (Fig. 6), and are consistent with the surgical therapeutic intent. During the follow-

Table 1

Descriptive measures of age and the longest follow-up by group and gender.

\begin{tabular}{|c|c|c|c|c|c|c|c|c|}
\hline \multirow[t]{2}{*}{ Group } & \multicolumn{4}{|c|}{ Age (years) } & \multicolumn{4}{|c|}{ Follow-up (months) } \\
\hline & mean & sd & $\min$ & $\max$ & mean & sd & $\min$ & $\max$ \\
\hline MMA: & & & & & & & & \\
\hline $\begin{array}{l}\text { female ( } 8) \\
\text { male (4) }\end{array}$ & $\begin{array}{l}33.3 \\
28.9\end{array}$ & $\begin{array}{l}20.2 \\
8.8\end{array}$ & $\begin{array}{l}15 \\
20\end{array}$ & $\begin{array}{l}67.0 \\
40.9\end{array}$ & $\begin{array}{l}12.8 \\
11.8\end{array}$ & $\begin{array}{l}1.6 \\
1.0\end{array}$ & $\begin{array}{l}11 \\
11\end{array}$ & $\begin{array}{l}15 \\
13\end{array}$ \\
\hline total (12) & 31.8 & 16.9 & 15 & 67.0 & 12.4 & 1.4 & 11 & 15 \\
\hline MMAd: & & & & & & & & \\
\hline female (15) & 28.8 & 15.0 & 15 & 58.6 & 14.7 & 6.3 & 11 & 36 \\
\hline male (5) & 32.6 & 10.8 & 19 & 44.2 & 20.0 & 10.6 & 11 & 38 \\
\hline total (20) & 29.8 & 13.9 & 15 & 58.6 & 16.1 & 7.6 & 11 & 38 \\
\hline
\end{tabular}

Table 2

Cephalometric comparison between groups before (T1) and for changes immediately following surgery (T2-T1).

\begin{tabular}{|c|c|c|c|c|c|c|c|c|c|c|}
\hline \multirow[t]{3}{*}{ measure } & \multicolumn{5}{|l|}{ T1 } & \multicolumn{5}{|l|}{$\mathrm{T} 2-\mathrm{T} 1$} \\
\hline & \multicolumn{2}{|l|}{ MMA } & \multicolumn{2}{|c|}{ MMAd } & \multirow[t]{2}{*}{$p$-value } & \multicolumn{2}{|l|}{ MMA } & \multicolumn{2}{|c|}{ MMAd } & \multirow[t]{2}{*}{$p$-value } \\
\hline & mean & sd & mean & sd & & mean & sd & mean & sd & \\
\hline SNA & 82.3 & 4.8 & 81.0 & 4 & 0.40 & 4.8 & 3.1 & 4.5 & 3.0 & 0.82 \\
\hline SNB & 77.4 & 3.5 & 75.2 & 5 & 0.18 & 5.2 & 2.2 & 4.8 & 3.3 & 0.71 \\
\hline SNGoMe & 37.3 & 8.4 & 39.8 & 9 & 0.45 & -4.3 & 5.1 & -4.5 & 5.0 & 0.92 \\
\hline FMA & 25.6 & 8.8 & 29.3 & 9 & 0.26 & -2.4 & 3.7 & -5.0 & 4.5 & 0.11 \\
\hline OPA & 15.8 & 6.3 & 19.1 & 5 & 0.12 & -6.7 & 5.5 & -6.7 & 4.1 & 0.99 \\
\hline AFH & 68.9 & 7.0 & 69.4 & 8 & 0.86 & -1.0 & 3.3 & -2.4 & 3.3 & 0.26 \\
\hline
\end{tabular}

Student's t-test and $\alpha=5 \%$, SNA - sella-nasion-A point angle, SNB - sella-nasion-B point angle, SN.GoMe - mandibular plane angle, FMA - Frankfort mandibular plane angle, OPA - occlusal plane angle, AFH - anterior facial height. up period (T3-T2), sagittal stability in the chin and B-point was similar for both groups (Table 5).

The proximal segment analysis showed that in the MMA group where patients were not submitted to articular disc repositioning, the condyles moved predominantly towards the superior and posterior immediately after surgery, as illustrated in Fig. 4. This displacement suggests that the presence of discs in their anatomical positions did not prevent the condyles from moving, showing the additional load that maxillomandibular advancement surgery exerts over the normal TMJs. In contrast, in individuals in the MMAd group, movement was towards the inferior and anterior (Fig. 5). These opposing directions are possibly due to the procedure of articular disc repositioning, which provides slight lowering of the condyles (anterior and inferior) to create space for disc repositioning (Wolford, 2003; Goncalves et al., 2013).

Another region assessed in the proximal segment was the inferior-posterior region of the ramus. In the MMA group, the

Table 3

Surgical changes (T2-T1) in groups 1 (MMA) and 2 (MMAd).

\begin{tabular}{|c|c|c|c|c|c|c|c|}
\hline \multirow[t]{2}{*}{ measure } & \multicolumn{2}{|l|}{ MMA } & \multicolumn{2}{|l|}{ MMAd } & \multicolumn{2}{|l|}{ Difference } & \multirow[t]{2}{*}{$p$-value } \\
\hline & mean $(\mathrm{mm})$ & sd & mean $(\mathrm{mm})$ & sd & mean $(\mathrm{mm})$ & se & \\
\hline Chin & 12.3 & 3.8 & 15.2 & 6.1 & -2.8 & 2.0 & 0.176 \\
\hline B-point & 8.7 & 2.6 & 9.4 & 3.8 & -0.7 & 1.3 & 0.584 \\
\hline Maxilla & 6.7 & 3.0 & 5.8 & 2.4 & 0.9 & 0.9 & 0.343 \\
\hline Lramus & 5.2 & 1.5 & 8.2 & 3.4 & -2.9 & 1.1 & $0.018 *$ \\
\hline Llatpole & 3.1 & 1.4 & 3.8 & 1.7 & -0.6 & 0.6 & 0.261 \\
\hline Lmedpole & 2.6 & 1.4 & 4.3 & 1.7 & -1.7 & 0.5 & $0.006 *$ \\
\hline Lsupcond & 3.2 & 1.4 & 4.6 & 2.0 & -1.4 & 0.6 & $0.037 *$ \\
\hline Lpostcond & 2.8 & 1.4 & 4.3 & 1.7 & -1.4 & 0.5 & $0.019 *$ \\
\hline Rramus & 5.3 & 1.7 & 7.9 & 3.4 & -2.6 & 1.1 & $0.027 *$ \\
\hline Rlatpole & 2.8 & 1.3 & 3.4 & 2.3 & -0.6 & 0.7 & 0.426 \\
\hline Rmedpole & 2.5 & 1.2 & 3.5 & 1.0 & -0.9 & 0.4 & $0.025 *$ \\
\hline Rsupcond & 3.1 & 1.7 & 3.6 & 1.2 & -0.4 & 0.5 & 0.384 \\
\hline Rpostcond & 2.9 & 1.3 & 3.1 & 1.2 & -0.2 & 0.4 & 0.576 \\
\hline
\end{tabular}

Student's t test and $\alpha=5 \%$.

$\mathrm{L}$ - left, $\mathrm{R}$ - right, latpole - condyle lateral pole, medpole - condyle medial pole supcond - condyle superior area, postcond - condyle posterior area. $\mathrm{p} \leq 0.05$, statistically different. 
Table 4

Adaptive responses and/or displacements (T3-T2) in groups 1 (MMA) and 2 (MMAd).

\begin{tabular}{|c|c|c|c|c|c|c|c|}
\hline \multirow[t]{2}{*}{ measure } & \multicolumn{2}{|l|}{ MMA } & \multicolumn{2}{|l|}{ MMAd } & \multicolumn{2}{|l|}{ Difference } & \multirow[t]{2}{*}{$p$-value } \\
\hline & mean $(\mathrm{mm})$ & sd & mean $(\mathrm{mm})$ & sd & mean $(\mathrm{mm})$ & se & \\
\hline Chin & 2.2 & 1.0 & 3.3 & 2 & -1.0 & 0.5 & 0.09 \\
\hline B point & 2.6 & 1.6 & 2.9 & 1 & -0.2 & 0.5 & 0.65 \\
\hline Maxilla & 2.7 & 1.2 & 3.5 & 2 & -0.8 & 0.5 & 0.12 \\
\hline Lramus & 4.5 & 1.9 & 4.0 & 2 & 0.4 & 0.6 & 0.53 \\
\hline Llatpole & 1.8 & 0.7 & 2.2 & 1 & -0.3 & 0.3 & 0.25 \\
\hline Lmedpole & 1.6 & 0.7 & 2.2 & 1 & -0.6 & 0.4 & 0.17 \\
\hline Lsupcond & 2.0 & 0.6 & 2.4 & 1 & -0.4 & 0.4 & 0.34 \\
\hline Lpostcond & 2.0 & 1.1 & 2.5 & 1 & -0.5 & 0.4 & 0.28 \\
\hline Rramus & 3.5 & 1.6 & 4.0 & 2 & -0.4 & 0.7 & 0.61 \\
\hline Rlatpole & 2.8 & 2.5 & 2.5 & 1 & 0.2 & 0.6 & 0.75 \\
\hline Rmedpole & 2.0 & 1.5 & 2.0 & 1 & 0.0 & 0.4 & 0.92 \\
\hline Rsupcond & 2.1 & 1.0 & 2.2 & 1 & -0.1 & 0.3 & 0.71 \\
\hline Rpostcond & 2.4 & 1.3 & 2.2 & 1 & 0.2 & 0.4 & 0.58 \\
\hline
\end{tabular}

Student's t test and $\alpha=5 \%$.

$\mathrm{L}=$ left side, $\mathrm{R}=$ right side; latpole - condyle lateral pole; medpole - condyle medial pole; supcond - condyle superior area; postcond - condyle posterior area.

surgical movements (T2-T1) displaced the ramus towards superior, anterior, and lateral with lateral roll (Table 5). In the MMAd group, the predominant dislocations were anterior with roll lateral (Table 5). These findings are similar to those of Carvalho et al. (2010) and De Paula et al (de Paula et al., 2013). When the distal segment is advanced, the increased width of the posterior region will be aligned with a narrower region of the proximal segment, leading to ramus and condyle torques. Therefore, T2-T3 visual analysis (Table 5) showed a displacement and/or remodelling in the opposite direction from that caused by surgery. In both groups the predominant direction of displacement of the ramus was towards the medial with medial roll. We believe that stretching of the medial pterygoid muscle is the main reason for those displacements (Dicker et al., 2012; Beukes et al., 2013). Besides, on the maxilla, the surgical procedures performed (T2-T1) were predominantly towards the anterior and superior. The mean values (Table 3) were higher than previously reported (Gonçalves et al., 2008), which is due to the method by which measurements were made - from the maximum values of the vectors rather than from a point determined from three different axes. For the postoperative adaptation (T3-T2) there were no differences between the two experimental groups. The maxillary adaptation that occurred towards the posterior can be attributed to remodelling of this area and the post-surgical orthodontic mechanics (Fig. 7).

Although the shape correspondence method provides a numerical maximal displacement, positive and negative values can indicate the results of movement in several different directions. We minimised these limitations by including a visual, qualitative method based on case-by-case assessment, using semi-transparent overlays that elucidated direction, displacement, remodelling, and amount of change (Table 5 and Figs. 4-7).

Another limitation of this study is that we could not assure that the repositioned discs were kept in place during the study period because post-surgical MRI data were not available. Clinical and

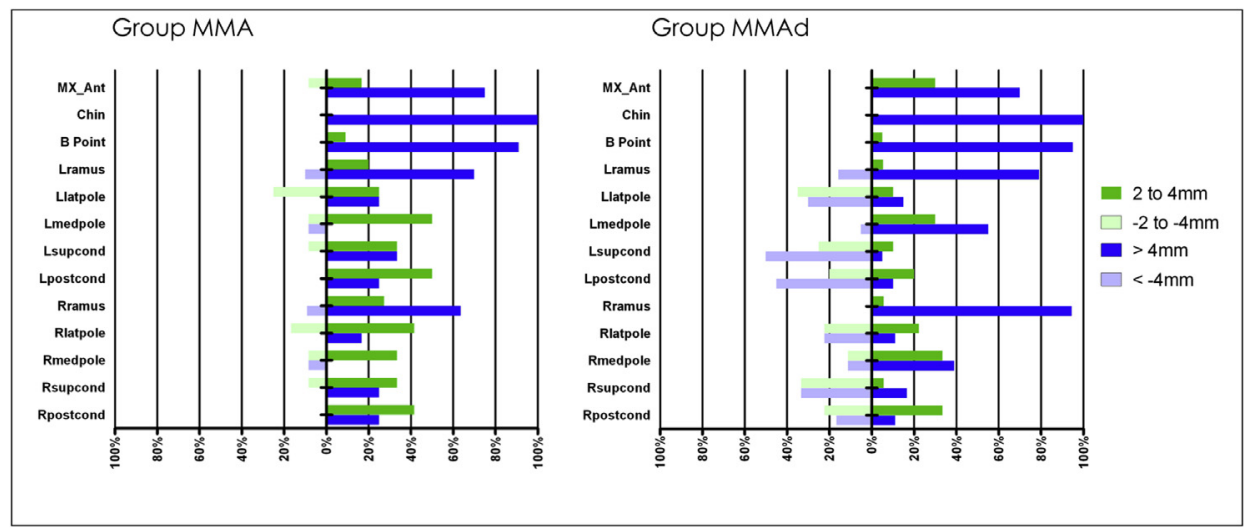

Fig. 2. Surgical displacement (T2-T1) and percentage of individuals among the groups MMA (A) and MMAd (B).

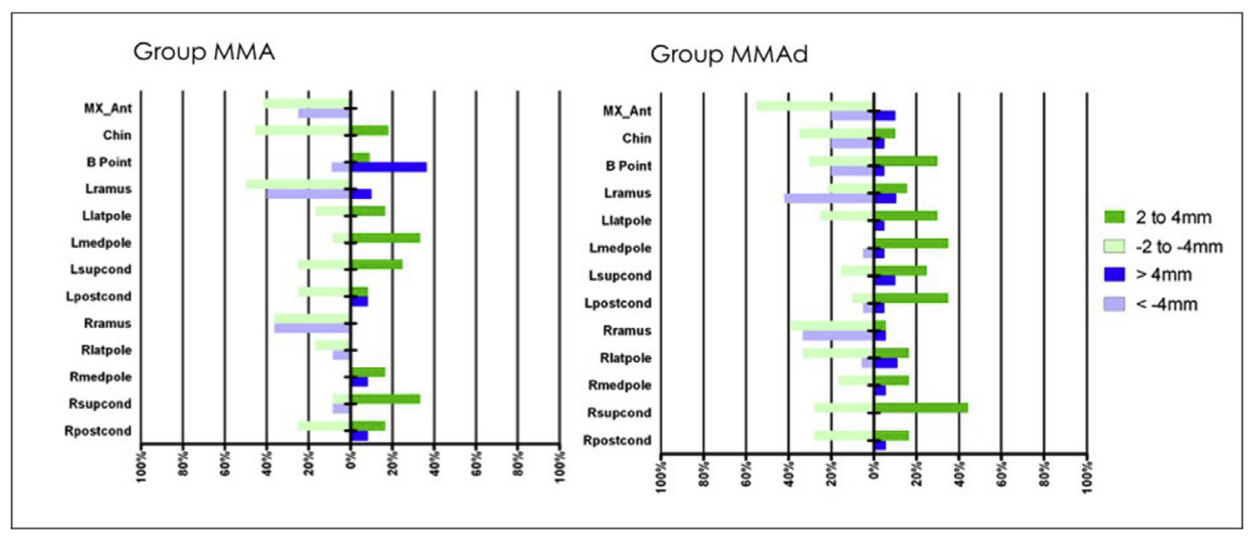

Fig. 3. Displacement and/or adaptive response (T3-T2) occurred in groups MMA (A) and MMAd (B). 
Table 5

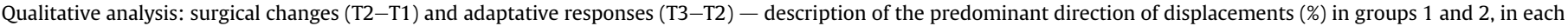
region of interest $(\mathrm{ROI})$.

\begin{tabular}{|c|c|c|c|c|}
\hline ROI & MMA (T2-T1) & MMAd (T2-T1) & MMA (T3-T2) & MMAd (T3-T2) \\
\hline Chin & Superior (83\%) Anterior (92\%) & $\begin{array}{l}\text { Superior (85\%) } \\
\text { Anterior }(100 \%)\end{array}$ & Inferior (60\%) Posterior (58\%) & Inferior (60\%) Posterior (60\%) \\
\hline B-point & Superior (75\%) Anterior (92\%) & $\begin{array}{l}\text { Superior }(85 \%) \\
\text { Anterior }(100 \%)\end{array}$ & Posterior (67\%) & Inferior (60\%) Posterior (55\%) \\
\hline Maxilla & Superior (100\%) Anterior (92\%) & $\begin{array}{l}\text { Superior }(90 \%) \\
\text { Anterior }(100 \%)\end{array}$ & Inferior (92\%) Posterior (100\%) & Inferior (90\%) Posterior (90\%) \\
\hline Rramus & $\begin{array}{l}\text { Superior (75\%) Anterior (75\%) } \\
\text { Lateral (58\%) Lateral roll (75\%) }\end{array}$ & $\begin{array}{l}\text { Anterior (55\%) Lateral (55\%) } \\
\text { Lateral roll }(75 \%)\end{array}$ & $\begin{array}{l}\text { Superior (75\%) Anterior (58\%) } \\
\text { Medial (75\%) Medial roll (67\%) }\end{array}$ & n.d.p. \\
\hline Lramus & $\begin{array}{l}\text { Superior (83\%) Anterior (83\%) } \\
\text { Lateral roll }(67 \%)\end{array}$ & Anterior (70\%) Lateral roll (80\%) & $\begin{array}{l}\text { Superior (67\%) Medial (75\%) } \\
\text { Medial roll (67\%) }\end{array}$ & Superior (80\%) Medial roll (65\%) \\
\hline Rlatpole & $\begin{array}{l}\text { Superior (75\%) Posterior }(92 \%) \\
\text { Medial roll (75\%) Yaw posterior (58\%) }\end{array}$ & Medial roll (65\%) & Medial (58\%) & Inferior (55\%) \\
\hline Llatpole & $\begin{array}{l}\text { Superior (58\%) Posterior (75\%) } \\
\text { Medial (67\%) Medial roll (75\%) }\end{array}$ & $\begin{array}{l}\text { Inferior (80\%) Medial (60\%) } \\
\text { Medial roll (85\%) }\end{array}$ & n.d.p. & Superior (55\%) Posterior (55\%) \\
\hline Rmedpole & $\begin{array}{l}\text { Inferior (58\%) Posterior (83\%) } \\
\text { Medial roll (83\%) }\end{array}$ & Inferior (65\%) Medial roll (60\%) & Medial (67\%) & Posterior (55\%) \\
\hline Lmedpole & $\begin{array}{l}\text { Inferior (58\%) Posterior (58\%) } \\
\text { Medial (67\%) Medial roll (83\%) }\end{array}$ & $\begin{array}{l}\text { Inferior (90\%) Anterior (55\%) } \\
\text { Medial (75\%) Medial roll (90\%) }\end{array}$ & Inferior (58\%) & n.d.p. \\
\hline Rsupcond & $\begin{array}{l}\text { Superior (67\%) Posterior (92\%) } \\
\text { Pitch up (67\%) }\end{array}$ & Inferior (70\%) Pitch up (55\%) & Superior (58\%) Anterior (58\%) & Posterior (55\%) \\
\hline Lsupcond & $\begin{array}{l}\text { Superior (58\%) Posterior (92\%) } \\
\text { Medial (58\%) Pitch up (67\%) }\end{array}$ & $\begin{array}{l}\text { Inferior (85\%) Medial (65\%) } \\
\text { Pitch up (70\%) }\end{array}$ & Superior (58\%) Posterior (58\%) & Superior (60\%) Posterior (55\%) \\
\hline Rpostcond & $\begin{array}{l}\text { Superior (67\%) Posterior (92\%) } \\
\text { Pitch up (67\%) }\end{array}$ & Inferior (70\%) & n.d.p. & Superior (55\%) Posterior (60\%) \\
\hline Lpostcond & $\begin{array}{l}\text { Superior (58\%) Posterior (92\%) } \\
\text { Medial (58\%) }\end{array}$ & Inferior (90\%) Medial (55\%) & Posterior (58\%) & Superior (60\%) Posterior (55\%) \\
\hline
\end{tabular}

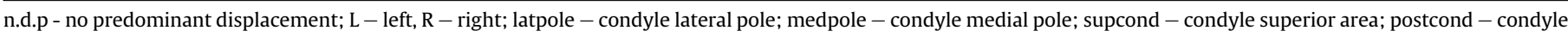
posterior area.
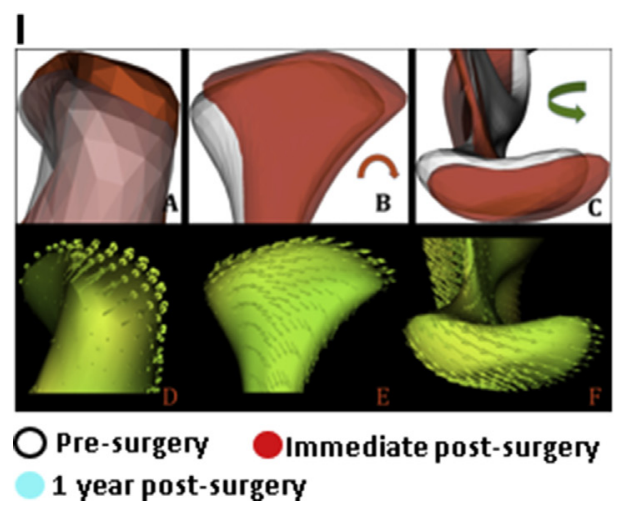
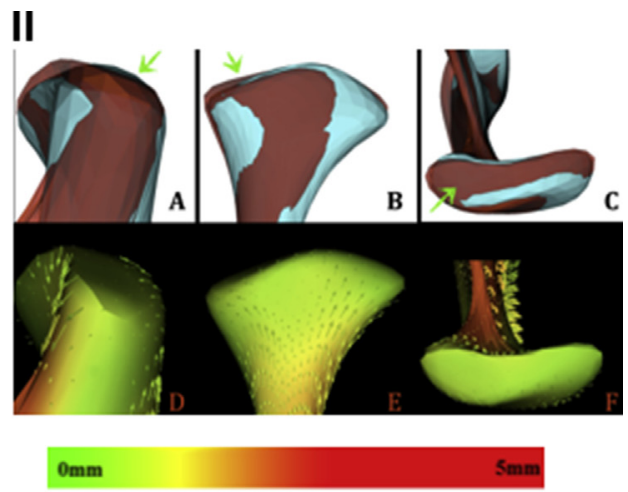

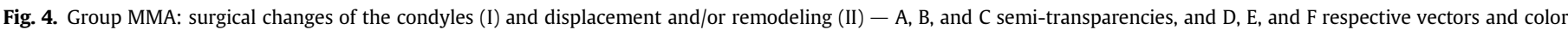
map. Green arrows showing displacement and/or resorption in the superior and posterior region.

I

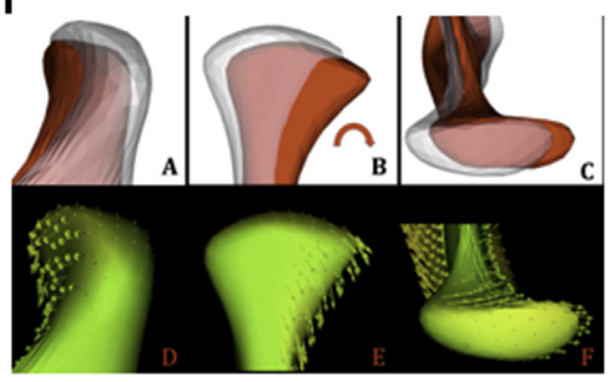

O Pre-surgery Immediate post-surgery

1 year post-surgery
II

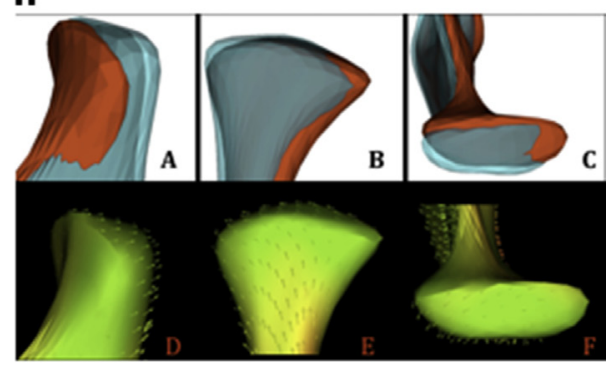

$0 \mathrm{~mm}$

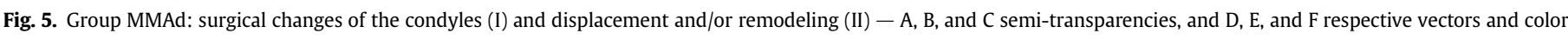
map. 


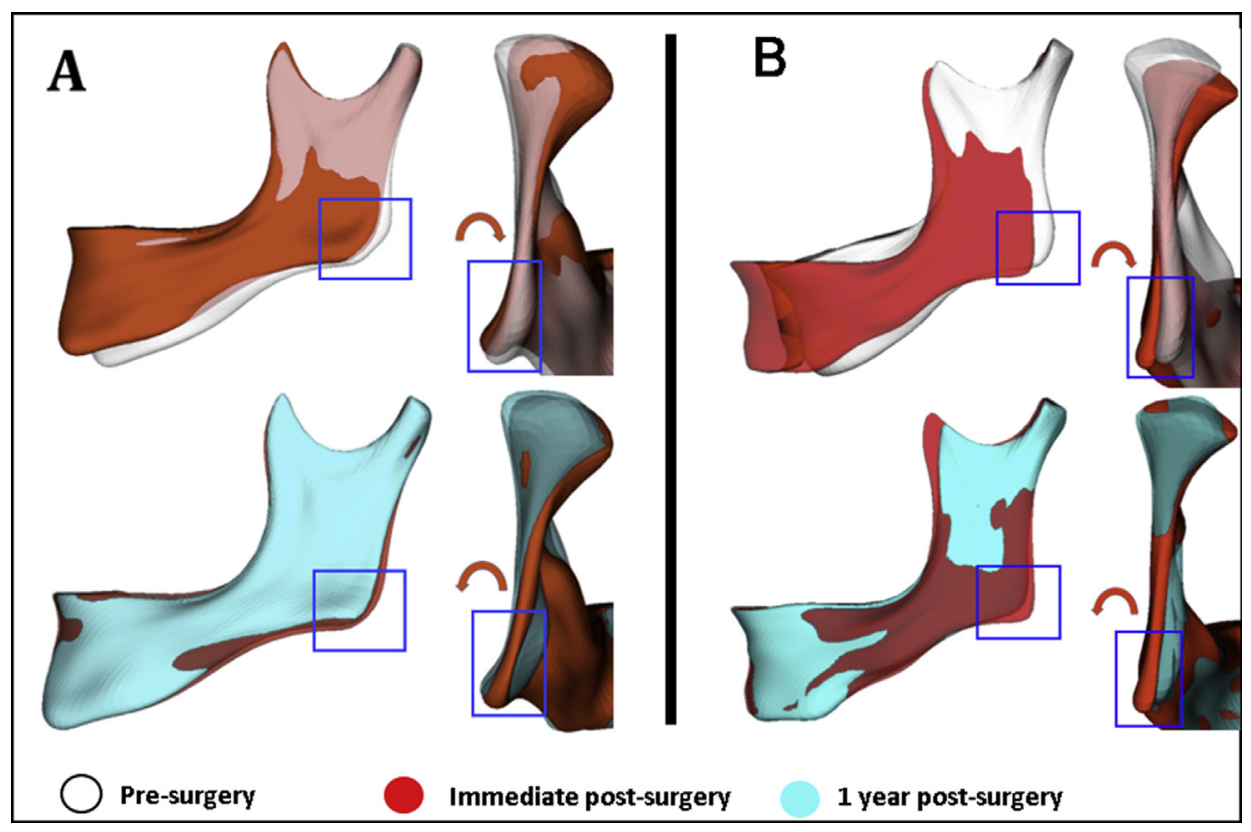

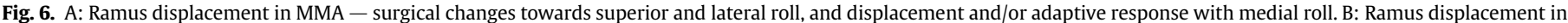
group MMAd - surgical changes towards anterior with lateral roll, and displacement and/or adaptive responses towards medial with medial roll.

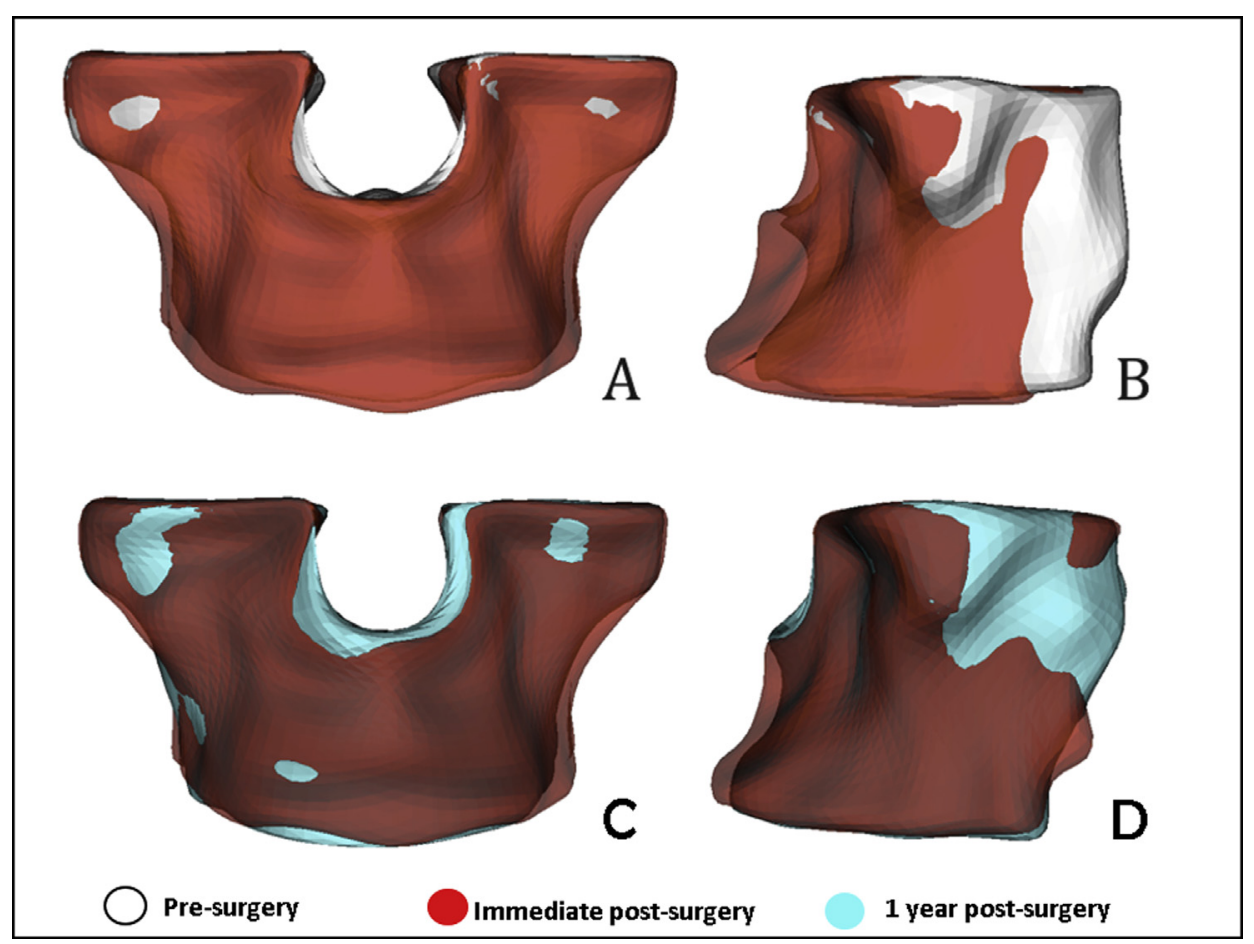

Fig. 7. Maxilla changes in group MMA, showing the advancement (A and B) and the relapse (C and D).

CBCT assessments suggested that the discs were kept in place throughout the entire study period.

\section{Conclusion}

Our findings supports our hypothesis, that there were no significant differences in skeletal stability (T3-T2) between the two groups. Maxillomandibular advancement surgeries with rotation of the occlusal plane showed good results for both groups immediately after surgery and at the longest follow-up. Condylar changes observed during surgery (T2-T1) were directly affected by the disc repositioning procedure.

\section{Disclaimer statements}

There is no potential conflict of interest. 


\section{Funding}

This research did not receive any specific grant from funding agencies in the public, commercial, or not-for-profit sectors.

\section{Acknowledgements}

Nothing to declare.

\section{References}

Ahmad M, Hollender L, Anderson Q, Kartha K, Ohrbach R, Truelove EL, et al: Research diagnostic criteria for temporomandibular disorders (RDC/TMD): development of image analysis criteria and examiner reliability for image analysis. Oral Surg Oral Med Oral Pathol Oral Radiol Endod 107(6): 844-860, 2009

Arnett GW, Milam SB, Gottesman L: Progressive mandibular retrusion-idiopathic condylar resorption. Part II. Am J Orthod Dentofac Orthop 110(2): 117-127, 1996

Beukes J, Reyneke JP, Becker PJ: Medial pterygoid muscle and stylomandibular ligament: the effects on postoperative stability. Int J Oral Maxillofac Surg 42(1): 43-48. https://doi.org/10.1016/j.ijom.2012.05.010, 2013

Borstlap WA, Stoelinga PJW, Hoppenreijs TJM, van't Hof MA: Stabilisation of sagittal split advancement osteotomies with miniplates: a prospective, multicentre study with two-year follow-up. Part III - condylar remodelling and resorption. Int J Oral Maxillofac Surg 33(7): 649-655. https://doi.org/10.1016/ j.ijom.2004.01.018, 2004

Carvalho FDAR, Cevidanes LHS, da Motta ATS, Almeida MADO, Phillips C: Threedimensional assessment of mandibular advancement 1 year after surgery. Am J Orthod Dentofacial Orthop 137(Suppl. 4): S53.e1-S53.e12. https://doi.org/ 10.1016/j.ajodo.2010.01.017, 2010

Cevidanes LHS, Bailey LJ, Tucker GR, Styner MA, Mol A, Phillips CL, et al: Superimposition of 3D cone-beam CT models of orthognathic surgery patients. Dentomaxillofacial Radiol 34(6): 369-375. https://doi.org/10.1259/dmfr/ 17102411, 2005

De Clerck H, Nguyen T, de Paula LK, Cevidanes L: Three-dimensional assessment of mandibular and glenoid fossa changes after bone-anchored class III intermaxillary traction. Am J Orthod Dentofac Orthop 142(1): 25-31. https://doi.org/ 10.1016/j.ajodo.2012.01.017, 2012

de Paula LK, Ruellas ACO, Paniagua B, Styner M, Turvey T, Zhu H, et al: One-year assessment of surgical outcomes in Class III patients using cone beam computed tomography. Int J Oral Maxillofac Surg 42(6): 780-789. https:// doi.org/10.1016/j.ijom.2013.01.002, 2013

Dicker GJ, Koolstra JH, Castelijns JA, Van Schijndel RA, Tuinzing DB: Positional changes of the masseter and medial pterygoid muscles after surgical mandibular advancement procedures: an MRI study. Int J Oral Maxillofac Surg 41(8): 922-929. https://doi.org/10.1016/j.ijom.2012.01.007, 2012

Franco AA, Cevidanes LHS, Phillips C, Rossouw PE, Turvey TA, Carvalho FDAR, et al: Almeida, MAO: long-term 3-dimensional stability of mandibular advancement surgery. J Oral Maxillofac Surg 71(9): 1588-1597. https://doi.org/10.1016 j.joms.2013.04.006, 2013

Gerressen M, Stockbrink G, Smeets R, Riediger D, Ghassemi A: Skeletal stability following bilateral sagittal split osteotomy (BSSO) with and without condylar positioning device. J Oral Maxillofac Surg 65(7): 1297-1302. https://doi.org 10.1016/j.joms.2006.10.026, 2007

Gill DS, El Maaytah M, Naini FB: Risk factors for post-orthognathic condylar resorption: a review. World J Orthod 9(1): 21-25, 2008

Gonçalves JR, Cassano DS, Wolford LM, Santos-Pinto A, Márquez IM: Postsurgical stability of counterclockwise maxillomandibular advancement surgery: affect of articular disc repositioning. J Oral Maxillofac Surg 66(4): 724-738. https:/| doi.org/10.1016/j.joms.2007.11.007, 2008

Goncalves JR, Wolford LM, Cassano DS, da Porciuncula G, Paniagua B, Cevidanes LH: Temporomandibular joint condylar changes following maxillomandibular advancement and articular disc repositioning. J Oral Maxillofac Surg 71(10). https://doi.org/10.1016/j.joms.2013.06.209, 2013 1759.e1-1759.e15

Mehra P, Wolford LM: The Mitek mini anchor for TMJ disc repositioning: surgica technique and results. Int J Oral Maxillofac Surg 30(6): 497-503. https:/ doi.org/10.1054/ijom.2001.0163, 2001

Nguyen T, Cevidanes L, Paniagua B, Zhu H, Koerich L, De Clerck H: Use of shape correspondence analysis to quantify skeletal changes associated with boneanchored Class III correction. Angle Orthod 84(2): 329-336. https://doi.org 10.2319/041513-288.1, 2013

Paniagua B, Cevidanes L, Walker D, Zhu H, Guo R, Styner M: Clinical application of SPHARM-PDM to quantify temporomandibular joint osteoarthritis. Comput Med Imaging Graph 35(5): 345-352. https://doi.org/10.1016/j.compmedimag.2010.11.012, 2011

Proffit WR, Turvey TA, Phillips C: The hierarchy of stability and predictability in orthognathic surgery with rigid fixation: an update and extension. Head Face Med 3: 21. https://doi.org/10.1186/1746-160X-3-21, 2007

Sidebottom AJ: Current thinking in temporomandibular joint management. Br J Ora Maxillofac Surg 47(2): 91-94. https://doi.org/10.1016/j.bjoms.2008.08.012, 2009

Wolford LM: Concomitant temporomandibular joint and orthognathic surgery. J Oral Maxillofac Surg 61(10): 1198-1204, 2003

Wolford LM, Reiche-Fischel O, Mehra P: Changes in temporomandibular joint dysfunction after orthognathic surgery. J Oral Maxillofac Surg 61(6): 655-660. https://doi.org/10.1053/joms.2003.50131, 2003 discussion 661

Yushkevich PA, Piven J, Hazlett HC, Smith RG, Ho S, Gee JC, et al: User-guided 3D active contour segmentation of anatomical structures: significantly improved efficiency and reliability. Neuroimage 31(3): 1116-1128. https://doi.org/10.1016 j.neuroimage.2006.01.015, 2006 